PROCEEDINGS OF THE

AMERICAN MATHEMATICAL SOCIETY

Volume 139, Number 9, September 2011, Pages 3327-3337

S 0002-9939(2011)10948-1

Article electronically published on March 9, 2011

\title{
ON THE LINEAR STABILITY OF KÄHLER-RICCI SOLITONS
}

\author{
STUART J. HALL AND THOMAS MURPHY \\ (Communicated by Jianguo Cao)
}

\begin{abstract}
We show that Kähler-Ricci solitons with $\operatorname{dim} H^{(1,1)}(M) \geq 2$ are linearly unstable. This extends the results of Cao-Hamilton-Ilmanen in the Kähler-Einstein case.
\end{abstract}

\section{INTRODUCTION}

A Ricci soliton is a complete Riemannian metric $g$, a vector field $X$ and a real number $c$ satisfying

$$
\operatorname{Ric}(g)+\mathcal{L}_{X} g-c g=0 .
$$

The soliton is called shrinking, steady or expanding if $c>0, c=0$ or $c<0$ respectively. If $X=\nabla f$ for a smooth function $f$ the soliton is said to be a gradient Ricci soliton with potential function $f$. If $X=0$ we recover the definition of an Einstein metric. Solitons are important in the theory of the Ricci flow as they occur as fixed points of the flow, up to the action of the diffeomorphism group. Perelman's groundbreaking observation 9 was that solitons are the critical points of a certain functional (the $\nu$-functional which we define below). An interesting question is whether the functional is locally maximised at these points (i.e. linearly stable) as this affects the behaviour of the Ricci flow nearby to a soliton. It is this question that we take up in this article for a special class of gradient shrinking solitons, namely Kähler-Ricci solitons. These are solitons on Fano manifolds which have the additional property that $g$ is a Kähler metric and the vector field $\nabla f$ is holomorphic.

The only known examples of compact Ricci solitons which are not products or Einstein are the Koiso-Cao soliton [7, 1], a $U(2)$-invariant soliton explicitly constructed on $\mathbb{C P}^{2} \sharp-\mathbb{C P}^{2}$ and a class of toric Kähler metrics constructed on Fano manifolds by Wang and Zhu [12.

The precise theorem we wish to prove is the following:

Theorem 1.1. If $(M, g, J)$ is a Kähler-Ricci soliton with $\operatorname{dim} H^{(1,1)}(M) \geq 2$, then the soliton is linearly unstable.

Received by the editors August 21, 2010.

2010 Mathematics Subject Classification. Primary 53C44; Secondary 53C25.

Key words and phrases. Ricci solitons, Perelman's $\nu$-functional, linear stability.

This work forms part of the first author's Ph.D thesis funded by the EPSRC. He would like to thank his advisor, Professor Simon Donaldson, for his comments and encouragement during the course of this work.

The second author was supported by an IRCSET postgraduate fellowship. The authors would also like to thank Professor Huai-Dong Cao for useful communications.

(C)2011 American Mathematical Society Reverts to public domain 28 years from publication 
Corollary 1.2. The Koiso-Cao soliton on $\mathbb{C P}^{2} \sharp-\mathbb{C P}^{2}$ and the Wang-Zhu soliton on $\mathbb{C P}^{2} \sharp-2 \mathbb{C P}^{2}$ are both linearly unstable.

This result generalises the result of Cao-Hamilton-Ilmanen who gave a simple argument in 4 for Kähler-Einstein metrics satisfying $\operatorname{dim} H^{(1,1)}(M) \geq 2$. The generalisation to Kähler-Ricci solitons is also suggested in their paper. We begin by recalling some facts about the $\nu$-functional, then proceed to compute the second variation of $\nu$ and define linear stability precisely. We finally show how one can construct unstable variations in a manner similar to 4 .

The question of linear stability for Kähler-Ricci solitons has also been considered by Tian and Zhu [10. They prove that if one considers variations in Kähler metrics in the fixed class $c_{1}(M)$, then the $\nu$-energy is maximised at a Kähler-Ricci soliton. There is also the recent work of Cao and Zhu [5], who also give a detailed calculation of the second variation of $\nu$.

\section{The Perelman $\nu$-FunCtional}

Throughout this paper $(M, g)$ will denote a closed Riemannian manifold. Most of the material here is contained in some form in [11] and [2] and, of course, was originally taken from [9. Perelman defined the following functional on triples $(g, f, \tau)$, where $g$ is a Riemannian metric, $f$ a smooth function and $\tau>0$ a constant.

Definition 2.1. Let $f$ be a smooth function on $M$ and $\tau>0$ a real number. Then the $W$-functional is given by

$$
W(g, f, \tau)=\int_{M}\left[\tau\left(R+|\nabla f|^{2}\right)+f-n\right](4 \pi \tau)^{-\frac{n}{2}} e^{-f} d V_{g},
$$

where $R$ is the scalar curvature of the metric $g$.

Some authors 10 absorb the constant $\tau$ into the other two terms, and it is not hard to see that $\mathcal{W}(g, f, \tau)=\mathcal{W}\left(\tau^{-1} g, f, 1\right)$. The $\mathcal{W}$-functional is also invariant under diffeomorphisms; i.e., $\mathcal{W}(g, f, \tau)=\mathcal{W}\left(\phi^{*} g, \phi^{*} f, \tau\right)$ for any diffeomorphism $\phi: M \rightarrow M$. Fix a compatibility condition for the triple $(g, f, \tau)$ by requiring

$$
\int_{M} \frac{e^{-f}}{(4 \pi \tau)^{\frac{n}{2}}} d V_{g}=1
$$

This leads to the definition of the $\nu$-functional:

Definition 2.2. Let $f$ be a smooth function on $M$ and $\tau>0$ a real number. Then the $W$-functional is given by

$$
\nu(g)=\inf \{\mathcal{W}(g, f, \tau):(g, f, \tau) \text { is compatible }\} .
$$

We will not comment on the existence theory except to say that for fixed $g$ there exists a $\tau>0$ and smooth $f$ that attain the infimum in the above definition. The pair $(f, \tau)$ satisfy the equations

$$
\tau\left(-2 \Delta f+|\nabla f|^{2}-R\right)-f+n+\nu=0 \text { and }(4 \pi \tau)^{-\frac{n}{2}} \int_{M} f e^{-f} d V_{g}=\frac{n}{2}+\nu .
$$

The first important result about the $\nu$-function is the following:

Theorem 2.3 (Perelman [9]). Let $g(t)$ be a family of Riemannian metrics on $M$ evolving via the Ricci flow. Then $\nu(g(t))$ is montone increasing, unless $g$ is a Ricci soliton, in which case $\nu$ is stationary. 
We also record the first variation of $\nu$ which makes it clear that stationary points of the functional are shrinking gradient Ricci solitons.

Theorem 2.4 (Perelman [9]). The first variation of $\nu$ in the direction $h, \mathcal{D}_{g} \nu(h)$, is given by

$$
-(4 \pi \tau)^{-\frac{n}{2}} \int_{M}\left\langle\tau(\operatorname{Ric}(g)+H e s s(f))-\frac{1}{2} g, h\right\rangle e^{-f} d V_{g} .
$$

\section{The SECOND VARIATION OF $\nu$}

The aim of this section is to give a self-contained proof of the second variation formula of $\nu$ at a Ricci soliton with potential function $f$. This is not new and has been known to experts for some time, but we include it here for completeness. We work with the scaled $L^{2}$-inner product on tensors

$$
\langle\cdot, \cdot\rangle_{f}=\int_{M}\langle\cdot, \cdot\rangle e^{-f} d V_{g}
$$

This inner product is adapted to the following operators:

$$
\operatorname{div}_{f}(h)=e^{f} \operatorname{div}\left(e^{-f} h\right) \text { and } \Delta_{f}(h)=\Delta(h)-\left(\nabla_{\nabla f} h\right),
$$

in the sense that

$$
\int_{M} \operatorname{div}_{f}(\alpha) e^{-f} d V_{g}=0 \text { and } \int_{M} \Delta_{f}(F) e^{-f} d V_{g}=0
$$

for any one-form $\alpha$ and function $F$. Obviously these reduce to the usual divergence and Laplacian when the metric is Einstein. The operator $\Delta_{f}$ is often referred to as the Bakry-Émery Laplacian. The sign convention for the curvature tensor we adopt is

$$
R(X, Y) Z=\nabla_{Y} \nabla_{X} Z-\nabla_{X} \nabla_{Y} Z+\nabla_{[X, Y]} Z
$$

and $R m(X, Y, W, Z)=g(R(X, Y) W, Z)$. In index notation we have $R\left(\partial_{i}, \partial_{j}, \partial_{k}, \partial_{l}\right)$ $=R m_{i j k l}$. For $h \in s^{2}\left(T M^{*}\right)$, define the symmetric curvature operator $R m(h, \cdot) \in$ $s^{2}\left(T M^{*}\right)$ by

$$
R m(h, \cdot)_{i j}=R_{k i l j} h^{k l} .
$$

We will also need the curvature operator on 2 -forms, usually denoted by $\mathcal{R}$ :

$$
\mathcal{R}: \Lambda^{2}(M) \rightarrow \Lambda^{2}(M), \quad \mathcal{R}(\sigma)_{i j}=R m_{i j k l} \sigma^{k l} .
$$

The convention for divergence we adopt is $\operatorname{div}(h)=\operatorname{tr}_{12}(\nabla h)$. The reader should note that this definition is the opposite sign to the divergence operator considered in [11. When restricted to forms we also have the codifferential $\delta$ which, with this convention, satisfies $\delta(\sigma)=-\operatorname{div}(\sigma)$. If we denote by $d i v_{f}^{*}$ the adjoint to $d i v_{f}$ with respect to the scaled $L^{2}$-inner product $\langle\cdot, \cdot\rangle_{f}$, then, as remarked in [3], div $v_{f}^{*}=d i v^{*}$. Here div ${ }^{*}$ is the adjoint to div with respect to the usual inner product on tensors.

Theorem 3.1 (Cao-Hamilton-Ilmanen). Let $g$ be a Ricci soliton with potential function $f$ satisfying Ric $(g)+H e s s(f)=\frac{1}{2 \tau} g$. For $h \in s^{2}\left(T M^{*}\right)$, consider variations $g(s)=g+s h$. Then the second variation of the $\nu$-energy is

$$
\left.\frac{d^{2}}{d s^{2}} \nu(g(s))\right|_{s=0}=\frac{\tau}{(4 \pi \tau)^{\frac{n}{2}}} \int_{M}\langle h, N h\rangle e^{-f} d V_{g},
$$

where $N$ is given by

$$
N(h)=\frac{1}{2} \Delta_{f}(h)+R m(h, \cdot)+\operatorname{div}_{f}^{*} \operatorname{div}_{f}(h)+\frac{1}{2} \operatorname{Hess}\left(v_{h}\right)-C(h, g) R i c .
$$


Here $v_{h}$ is the solution of

$$
\Delta_{f}\left(v_{h}\right)+\frac{1}{2 \tau} v_{h}=\operatorname{div}_{f} \operatorname{div}_{f}(h),
$$

and $C(h, g)$ is a constant depending upon $h$ and $g$.

Remark 3.2. This theorem was first stated in [3] but with an error in the term $C(h, g)$. This has subsequently been corrected in the recent work of Cao and Zhu [5]. They find that

where $R$ is the scalar curvature of $g$.

$$
C(h, g)=\frac{\int_{M}\langle R i c, h\rangle e^{-f} d V_{g}}{\int_{M} R e^{-f} d V_{g}}
$$

Definition 3.3. A soliton is linearly stable if the operator $N$ is non-positive-definite and linearly unstable otherwise.

We now collect some formulae for how various geometric quantities vary through a family of Riemannian metrics $g(t)$ evolving via $\frac{\partial g}{\partial t}=h$, where $h \in s^{2}\left(T M^{*}\right)$. Much of what is required is explained extremely well in [11, and so many proofs are omitted.

Lemma 3.4 (Propositions 2.3.7 and 2.3.9 in [11]). Let $g(s)=g+$ sh be a family of Riemannian metrics on $M$. Then

$$
\begin{aligned}
\left.\frac{d}{d s} \operatorname{Ric}(g(s))\right|_{s=0} & =\frac{1}{2} \nabla^{*} \nabla(h)-\operatorname{Rm}(h, \cdot)+\frac{1}{2}(\operatorname{Ric} \cdot h+h \cdot \operatorname{Ric}) \\
& -\operatorname{div}^{*} \operatorname{div}(h)-\frac{1}{2} \operatorname{Hess}(\operatorname{tr}(h)) .
\end{aligned}
$$

Let $R(s)=\operatorname{tr}_{g(s)} \operatorname{Ric}(g(s))$ be the scalar curvature of $g(s)$. Then

$$
\left.\frac{d}{d s} R(s)\right|_{s=0}=-\langle h, R i c\rangle+\operatorname{div} \operatorname{div}(h)-\Delta(\operatorname{tr}(h)) .
$$

It is necessary to know how geometric quantities associated to functions $f$ : $(-\epsilon, \epsilon) \times M \rightarrow \mathbb{R}$ vary. The convention we adopt is that $\Delta f=\operatorname{tr}(\operatorname{Hess}(f))=-\delta d f$, so the Laplacian has negative eigenvalues.

Lemma 3.5 (Prop. 2.3.10 in [11]). Let $g(s)=g+$ sh be a family of Riemannian metrics on $M$ and let $f(s, x):(-\epsilon, \epsilon) \times M \rightarrow \mathbb{R}$ be a family of smooth functions. Then

$$
\begin{aligned}
\left.\frac{d}{d s} \Delta(f)\right|_{s=0} & =\Delta_{g}(\dot{f})-\frac{1}{2}\langle d(\operatorname{tr}(h)), d f\rangle-\langle h, \operatorname{Hess}(f)\rangle-\langle\operatorname{div}(h), d f\rangle, \\
\left.\frac{d}{d s}|\nabla f|^{2}\right|_{s=0} & =-h(\nabla f, \nabla f)+2\langle\nabla f, \nabla \dot{f}\rangle .
\end{aligned}
$$

The final object whose variation must be computed is the Hessian of the functions $f(s, x)$.

Lemma 3.6. Let $g(s)=g+$ sh be a family of Riemannian metrics on $M$ and let $f(s, x):(-\epsilon, \epsilon) \times M \rightarrow \mathbb{R}$ be a family of smooth functions. Then

$$
\left.\frac{d}{d s} \operatorname{Hess}(f)\right|_{s=0}=\operatorname{Hess}(\dot{f})+\frac{1}{2}(\operatorname{Hess}(f) \cdot h+h \cdot H \operatorname{ess}(f))+\frac{1}{2}\left(\nabla_{\nabla f} h\right)+\operatorname{div}^{*}(h(\nabla f, \cdot)) \text {. }
$$


Proof. The Hessian of a function $f$ is given by $\operatorname{Hess}(X, Y)=g\left(\nabla_{X} \nabla f, Y\right)$. Hence

$$
\begin{aligned}
\frac{d}{d s} \operatorname{Hess}(f)(X, Y) & =h\left(\nabla_{X} \nabla f, Y\right)+g\left(\frac{d}{d s}\left(\nabla_{X} \nabla f\right), Y\right) \\
& =h\left(\nabla_{X} \nabla f, Y\right)+g\left(\frac{d}{d s}\left(\nabla_{X}\right) \nabla f, Y\right)+g\left(\nabla_{X} \frac{d}{d s} \nabla f, Y\right) .
\end{aligned}
$$

By Proposition 2.3.1 in [11,

$$
g\left(\frac{d}{d s}\left(\nabla_{X}\right) \nabla f, Y\right)=\frac{1}{2}\left[\left(\nabla_{\nabla f} h\right)(X, Y)+\left(\nabla_{X} h\right)(Y, \nabla f)-\left(\nabla_{Y} h\right)(X, \nabla f)\right] .
$$

We also have

$$
\begin{aligned}
g\left(\nabla_{X} \frac{d}{d s} \nabla f, Y\right)+h\left(\nabla_{X} \nabla f, Y\right) & =g\left(\nabla_{X} \nabla \dot{f}, Y\right)-\left(\nabla_{X} h\right)(Y, \nabla f) \\
& =H e s s(\dot{f})(X, Y)-\left(\nabla_{X} h\right)(Y, \nabla f) .
\end{aligned}
$$

Hence the variation is given by

$$
\begin{aligned}
& \operatorname{Hess}(\dot{f})(X, Y)+\frac{1}{2}\left(\nabla_{\nabla f} h\right)(X, Y)+\frac{1}{2}\left[h\left(\nabla_{X} \nabla f, Y\right)+h\left(\nabla_{Y} \nabla f, X\right)\right] \\
& -\frac{1}{2}\left[\left(\nabla_{X} h(\nabla f, \cdot)\right)(Y)+\left(\nabla_{Y} h(\nabla f, \cdot)\right)(X)\right]
\end{aligned}
$$

and the result follows.

Lemma 3.7. For $h \in s^{2}\left(T M^{*}\right)$, then

$$
\operatorname{div}_{f} \operatorname{div}_{f}(h)=\operatorname{div} \operatorname{div}(h)+h(\nabla f, \nabla f)-2\langle d f, \operatorname{div}(h)\rangle-\langle h, \operatorname{Hess}(f)\rangle .
$$

Proof. From the definition of $\operatorname{div}_{f}$ we have $\operatorname{div}_{f}(h)(\cdot)=\operatorname{div}(h)(\cdot)-h(\nabla f, \cdot)$, so we need to compute

$$
\begin{aligned}
\operatorname{div}_{f}(\operatorname{div}(h)(\cdot)-h(\nabla f, \cdot)) & =e^{f} \operatorname{div}\left(e^{-f}(\operatorname{div}(h)(\cdot)-h(\nabla f, \cdot))\right. \\
& =\operatorname{div} \operatorname{div}(h)-\operatorname{div}(h)(\nabla f)-\operatorname{div}(h(\nabla f, \cdot))+h(\nabla f, \nabla f) .
\end{aligned}
$$

The term $\operatorname{div}(h(\nabla f, \cdot))=\operatorname{div}(h)(\nabla f)+\langle h, \operatorname{Hess}(f)\rangle$, and so

$$
\operatorname{div}_{f} \operatorname{div}_{f}(h)=\operatorname{div} \operatorname{div}(h)+h(\nabla f, \nabla f)-2\langle d f, \operatorname{div}(h)\rangle-\langle h, H e s s(f)\rangle .
$$

If the soliton varies by $\delta g=h$, then this induces a variation in the pair $(f, \tau)$, which is denoted by $(\delta f, \delta \tau)$.

Lemma 3.8. Let $(g, f, \tau)$ describe a Ricci soliton and consider a variation $g(s)=$ $g+$ sh inducing variations $(\delta f, \delta \tau)$ in $f$ and $\tau$. If $v_{h}=\left(\operatorname{tr}(h)-2 \delta f-\frac{2 \delta \tau}{\tau}(f-\nu)\right)$, then $v_{h}$ satisfies

and

$$
\Delta_{f}\left(v_{h}\right)+\frac{v_{h}}{2 \tau}=\operatorname{div}_{f} d i v_{f}(h)
$$

$$
\int_{M}\left(-\frac{n \delta \tau}{2 \tau} f+\delta f(1-f)+f \frac{1}{2} \operatorname{tr}(h)\right) e^{-f} d V_{g}=0 .
$$

Proof. Consider the variation in the equation

$$
\tau\left(-2 \Delta f+|\nabla f|^{2}-R\right)-f+n+\nu=0,
$$

which yields

$$
\delta \tau\left(-2 \Delta f+|\nabla f|^{2}-R\right)+\tau\left(-2 \delta(\Delta f)+\delta|\nabla f|^{2}-\delta R\right)-\delta f+\delta \nu=0 .
$$


As we are at a soliton, $\delta \nu=0$ and $R=\frac{n}{2 \tau}-\Delta f$. Hence

$$
-2 \Delta f+|\nabla f|^{2}-R=-\Delta f+|\nabla f|^{2}-\frac{n}{2 \tau}=-\Delta_{f} f-\frac{n}{2 \tau} .
$$

Lemmas 3.4 and 3.5 imply that

$$
\delta(-2 \Delta f)=\Delta(-2 \delta f)+\langle\nabla \operatorname{tr}(h), \nabla f\rangle+2\langle h, \operatorname{Hess}(f)\rangle+2\langle\operatorname{div}(h), d f\rangle
$$

and

$$
\begin{aligned}
-\delta R & =\langle h, R i c\rangle-\operatorname{div} \operatorname{div}(h)+\Delta(\operatorname{tr}(h)) \\
& =-\langle h, H e s s\rangle+\frac{1}{2 \tau} \operatorname{tr}(h)-\operatorname{div} \operatorname{div}(h)+\Delta(\operatorname{tr}(h)) .
\end{aligned}
$$

This yields

$$
\begin{aligned}
& \frac{\delta \tau}{\tau}\left(-2 \Delta f+|\nabla f|^{2}-R\right)+\Delta(\operatorname{tr}(h)-2 \delta f)+\langle\nabla(\operatorname{tr}(h)-2 \delta f), \nabla f\rangle \\
& \quad+\frac{1}{2 \tau}(\operatorname{tr}(h)-2 \delta f)=\operatorname{div} \operatorname{div}(h)+h(\nabla f, \nabla f)-\langle h, H e s s\rangle-2\langle\operatorname{div}(h), d f\rangle .
\end{aligned}
$$

The left hand side is $\operatorname{div}_{f} \operatorname{div}_{f}(h)$, by Lemma 3.8, and the right hand side may be written as

$$
\frac{\delta \tau}{\tau}\left(-2 \Delta f+|\nabla f|^{2}-R\right)+\Delta_{f}(\operatorname{tr}(h)-2 \delta f)+\frac{1}{2 \tau}(\operatorname{tr}(h)-2 \delta f) .
$$

At a Ricci soliton, the scalar curvature $R$ is given by $R=-\Delta f+\frac{n}{2 \tau}$, so

$$
\Delta_{f}(f)=\Delta(f)-|\nabla f|^{2}=2 \Delta(f)-|\nabla f|^{2}+R-\frac{n}{2 \tau}=\frac{-f}{\tau}+\frac{n}{2 \tau}+\frac{\nu}{\tau},
$$

whence

$$
\Delta_{f} f+\frac{f}{2 \tau}=-\frac{f}{2 \tau}+\frac{n}{2 \tau}+\frac{\nu}{\tau} .
$$

Setting $\tilde{f}=-\frac{2 \delta \tau}{\tau}(f-\nu)$,

$$
\Delta_{f}(\tilde{f})+\frac{\tilde{f}}{2 \tau}=\frac{\delta \tau}{\tau}\left(\frac{f}{\tau}\right)-\frac{2 \delta \tau}{\tau}\left(\frac{n}{2 \tau}+\frac{\nu}{\tau}\right)+\frac{\delta \tau \nu}{\tau^{2}}=\frac{\delta \tau}{\tau}\left(-2 \Delta f+|\nabla f|^{2}-R\right) .
$$

Now letting $v_{h}=\operatorname{tr}(h)-2 \delta f-2 \frac{\delta \tau(f-\nu)}{\tau}$,

$$
\Delta_{f}\left(v_{h}\right)+\frac{v_{h}}{2 \tau}=\operatorname{div}_{f} \operatorname{div}_{f}(h) .
$$

The second equation is simply the variation in the equation

$$
(4 \pi \tau)^{\frac{-n}{2}} \int_{M} f e^{-f} d V_{g}=\frac{n}{2}+\nu
$$

We proceed to the proof of Theorem 3.1

Proof. The first variation is given by

$$
\left.\frac{d}{d s} \nu(g)\right|_{s=0}=(4 \pi \tau)^{-\frac{n}{2}} \int_{M}-\left\langle h, \tau(\operatorname{Ric}+\operatorname{Hess}(f))-\frac{1}{2} g\right\rangle e^{-f} d V_{g},
$$

so it is sufficient to compute the variation in the term

$$
\tau(\operatorname{Ric})+\operatorname{Hess}(f))-\frac{1}{2} g .
$$

This is given by

$$
\delta \tau(\operatorname{Ric}(g)+H e s s(f))-\frac{1}{2} h+\tau(\delta \operatorname{Ric}+\delta H e s s(f)) .
$$


Using the previous results,

$$
\delta R i c=\frac{1}{2} \nabla^{*} \nabla(h)-R m(h, \cdot)+\frac{1}{2}(R i c \cdot h+h \cdot R i c)-\operatorname{div}{ }^{*} \operatorname{div}(h)-\frac{1}{2} \operatorname{Hess}(\operatorname{tr}(h)),
$$

which, using the fact we are at a soliton, yields

$$
\begin{aligned}
\delta R i c= & \frac{1}{2} \nabla^{*} \nabla(h)-\operatorname{Rm}(h, \cdot)-\frac{1}{2}(\operatorname{Hess}(f) \cdot h+h \cdot \operatorname{Hess}(f))+\frac{h}{2 \tau} \\
& -\operatorname{div}^{*} \operatorname{div}(h)-\frac{1}{2} \operatorname{Hess}(\operatorname{tr}(h)) .
\end{aligned}
$$

The variation in the Hessian is given by

$$
\operatorname{Hess}(\dot{f})+\frac{1}{2}(\operatorname{Hess}(f) \cdot h+h \cdot \operatorname{Hess}(f))+\frac{1}{2}\left(\nabla_{\nabla f} h\right)+\operatorname{div}^{*}(h(\nabla f, \cdot)) .
$$

Putting this together, the variation is given by

$$
\begin{aligned}
& \delta \tau \operatorname{Ric}(g)-\frac{\tau}{2}\left(\Delta(h)-\left(\nabla_{\nabla f} h\right)\right)-\tau \operatorname{Rm}(h, \cdot)-\tau \operatorname{div}^{*}(\operatorname{div}(h)-h(\nabla f, \cdot)) \\
& +\frac{\tau}{2} \operatorname{Hess}\left(\frac{2 \delta \tau}{\tau} f+2 \delta f-\operatorname{tr}(h)\right)
\end{aligned}
$$

which may be rewritten as

$$
=\delta \tau \operatorname{Ric}(g)+\tau\left(-\frac{1}{2} \Delta_{f}(h)-\operatorname{Rm}(h, \cdot)-\operatorname{div}_{f}^{*} \operatorname{div}_{f}(h)-\frac{1}{2} \operatorname{Hess}\left(v_{h}\right)\right) .
$$

The result follows on taking $\frac{\delta \tau}{\tau}=C(h, g)$.

Taking $f$ constant recovers:

Corollary 3.9 (Einstein Case [4]). Let $g$ be an Einstein metric with Ric $(g)=\frac{1}{2 \tau} g$. For $h \in s^{2}\left(T M^{*}\right)$, consider variations $g(s)=g+s h$. Then the second variation of the $\nu$-energy at $g$ is

$$
\left.\frac{d^{2}}{d s}\right|_{s=0} \nu(g(s))=\frac{\tau}{\operatorname{vol}(g)} \int_{M}\langle h, N h\rangle d V_{g}
$$

where $N$ is given by

$N(h)=-\frac{1}{2} \nabla^{*} \nabla(h)+R m(h, \cdot)+\operatorname{div} v^{*} \operatorname{div}(h)+\frac{1}{2} \operatorname{Hess}\left(v_{h}\right)-\frac{g}{2 n \tau \operatorname{vol}(g)} \int_{M} \operatorname{tr}(h) d V_{g}$.

Here $v_{h}$ is the solution of

$$
\Delta\left(v_{h}\right)+\frac{1}{2 \tau} v_{h}=\operatorname{div} \operatorname{div}(h)
$$

\section{The Instability of KÄHLER-RicCI SOLITONS}

We first establish the following simple lemma comparing curvature operators on a Kähler manifold:

Lemma 4.1. Let $(M, g, J)$ be a Kähler manifold. If $\sigma \in \mathcal{A}^{(1,1)}(M)$, then $2 \operatorname{Rm}\left(\sigma_{J}, \cdot\right)$ $-\mathcal{R}(\sigma)_{J}=0$. 
Proof. We calculate using an adapted orthonormal basis $\left\{e_{i}, J e_{i}\right\}$. From the definition of $\mathcal{R}$, the Bianchi identity and the $J$-invariance of the curvature tensor,

$$
\begin{aligned}
\mathcal{R}(\sigma)(X, J Y) & =\sum_{i, j} R\left(e_{i}, e_{j}, X, J Y\right) \sigma\left(e_{i}, e_{j}\right) \\
& =-\sum_{i, j}\left(R\left(X, e_{i}, e_{j}, J Y\right)+R\left(e_{j}, X, e_{i}, J Y\right)\right) \sigma\left(e_{i}, e_{j}\right) \\
& =\sum_{i, j}\left(R\left(X, e_{i}, J e_{j}, Y\right)+R\left(e_{j}, X, J e_{i}, Y\right)\right) \sigma\left(e_{i}, e_{j}\right) \\
& =\sum_{i, j}-2 R\left(e_{i}, X, J e_{j}, Y\right) \sigma\left(e_{i}, e_{j}\right) \\
& =\sum_{i, j} 2 R\left(e_{i}, X, J e_{j}, Y\right) \sigma\left(e_{i}, J\left(J e_{j}\right)\right) \\
& =2 R m\left(\sigma_{J}, \cdot\right)(X, Y) .
\end{aligned}
$$

The proof of the main result will be modelled on the Kähler-Einstein case, which is outlined in 4 .

Proposition 4.2. Let $(M, g, J)$ be a Kähler-Einstein manifold with $\operatorname{dim} H^{(1,1)}(M)$ $\geq 2$. Then $g$ is linearly unstable.

Proof. Choose a trace-free harmonic $(1,1)$-form $\sigma \in \mathcal{H}^{(1,1)}(M)$ that induces a perturbation $\sigma_{J}=\sigma(\cdot, J \cdot)$. As the complex structure is parallel, $\operatorname{div}\left(\sigma_{J}\right)=0$ and $\left(\nabla^{*} \nabla \sigma_{J}\right)=\left(\nabla^{*} \nabla \sigma\right)_{J}$. We can use a Weitzenbock formula on 2-forms to compare the rough Laplacian with the Hodge Laplacian $\Delta_{H}=-(d+\delta)^{2}$. The Weitzenbock formula for an Einstein metric with Einstein constant $\frac{1}{2 \tau}$ is

$$
-\Delta_{H}=\nabla^{*} \nabla-\mathcal{R}+\frac{1}{\tau} i d
$$

where $\mathcal{R}$ is the curvature operator for 2 -forms. Our formula for the variation becomes

$$
\begin{aligned}
2 N\left(\sigma_{J}\right) & =\Delta_{H}(\sigma)_{J}-\mathcal{R}(\sigma)_{J}+\frac{1}{\tau} \sigma_{J}+2 \operatorname{Rm}\left(\sigma_{J}, \cdot\right)+\operatorname{Hess}\left(v_{\sigma_{J}}\right) \\
& =-\mathcal{R}(\sigma)_{J}+\frac{1}{\tau} \sigma_{J}+2 \operatorname{Rm}\left(\sigma_{J}, \cdot\right)+\operatorname{Hess}\left(v_{\sigma_{J}}\right) .
\end{aligned}
$$

It follows from Equation 3.1 that the function $v_{\sigma_{J}}$ is an eigenfunction of the Laplacian. Recall that the first non-zero eigenvalue of the Laplacian on an Einstein manifold with Einstein constant $c>0$ satisfies

$$
\lambda_{1} \leq \frac{-n}{n-1} c
$$

by the famous Lichnerowicz bound. Hence $v_{\sigma_{J}}=0$. From Lemma 4.1 $2 R m\left(\sigma_{J}, \cdot\right)$ $-\mathcal{R}(\sigma)_{J}=0$, so we have exhibited an eigentensor for $N$ with eigenvalue $\frac{1}{2 \tau}$. Hence metrics of this type are unstable.

Considering the divergence operator $\delta: \Omega(M)^{k} \rightarrow \Omega(M)^{k-1}$ as the adjoint of the exterior derivative on forms, then, with our conventions, $\operatorname{div}(\sigma)=-\delta(\sigma)$ for any $k$-form $\sigma$. Set

$$
\delta_{f}(\sigma)=e^{f} \delta\left(e^{-f} \sigma\right)=\delta(\sigma)+\iota_{\nabla f} \sigma \text { and } \Delta_{f, H}(\sigma)=-\left(d \delta_{f}+\delta_{f} d\right)(\sigma) .
$$


We will refer to $\Delta_{f, H}$ as the twisted Laplacian. Forms $\sigma$ satisfying $\Delta_{f, H}(\sigma)=0$ are said to be twisted harmonic forms. Modifications of the Hodge Laplacian similar to this are also found in the seminal work of Witten [13] on the Morse inequalities. The following lemma gives some crucial properties of this Laplacian:

Lemma 4.3. The operator $\Delta_{f, H}$ has the following properties:

(1) $\Delta_{f, H}=\Delta_{H}-\mathcal{L}_{\nabla f}$.

(2) $\Delta_{f, H}$ preserves the decomposition into $(p, q)$-forms.

(3) $\Delta_{f, H}$ satisfies a Weitzenbock identity for 2 -forms $\sigma$ :

$$
\Delta_{f}(\sigma)-\Delta_{f, H}(\sigma)=-\mathcal{R}(\sigma)+\frac{1}{\tau} \sigma .
$$

Proof. From the definition we have

$$
\Delta_{f, H}(\sigma)=\Delta_{H}(\sigma)-\left(d \circ \iota_{\nabla f}+\iota_{\nabla f} \circ d\right) \sigma .
$$

Using Cartan's magic formula this yields

$$
\Delta_{f, H}(\sigma)=\Delta_{H}(\sigma)-\mathcal{L}_{\nabla f}(\sigma) .
$$

The second claim follows from the fact that $\nabla f$ is a holomorphic vector field. For the Weitzenbock formula recall that for 2-forms

$$
\begin{aligned}
-\nabla^{*} \nabla(\sigma)-\Delta_{H}(\sigma) & =-\mathcal{R}(\sigma)+\text { Ric } \cdot \sigma+\sigma \cdot \text { Ric } \\
& =-\mathcal{R}(\sigma)-(\operatorname{Hess}(f) \cdot \sigma+\sigma \cdot \operatorname{Hess}(f))+\frac{1}{\tau} \sigma,
\end{aligned}
$$

where $\mathcal{R}$ is the curvature operator for 2 -forms. A simple computation yields

$$
\mathcal{L}_{\nabla f}(\sigma)-\left(\nabla_{\nabla f}\right)(\sigma)=(\operatorname{Hess}(f) \cdot \sigma+\sigma \cdot \operatorname{Hess}(f)) .
$$

Hence

$$
\left(\Delta_{f}-\Delta_{f, H}\right)(\sigma)=-\mathcal{R}(\sigma)+\frac{1}{\tau} \sigma .
$$

To conclude, the proof of Theorem 1.1 is presented.

Proof. The first step is to choose a twisted harmonic form $\sigma \in H^{(1,1)}(M)$. If we denote the space of twisted harmonic forms by $\mathcal{H}_{f}$, then we have the natural map

$$
\pi: \mathcal{H}_{f} \rightarrow H^{2}(M) \text { given by } \pi(\sigma)=[\sigma] .
$$

The proof that this map is an isomorphism follows that of the usual Hodge theorem except that the 'energy' in our case is given by

$$
\int_{M}\|\sigma\|^{2} e^{-f} d V_{g}
$$

The twisted Laplacian also preserves the $(p, q)$-decomposition of forms, so we get the required isomorphism of vector spaces

$$
\mathcal{H}_{f}^{(1,1)} \cong H^{(1,1)}(M) .
$$

The hypothesis on the dimension of $H^{(1,1)}(M)$ means that there exist $\theta_{1}, \theta_{2} \in \mathcal{H}_{f}^{(1,1)}$ and $\lambda, \mu \in \mathbb{R}$, not both zero, satisfying

$$
\left\langle\lambda \theta_{1}+\mu \theta_{2}, \rho\right\rangle_{f}=\int_{M}\left\langle\lambda \theta_{1}+\mu \theta_{2}, \rho\right\rangle e^{-f} d V_{g}=0
$$


where $\rho$ is the Ricci form. Choosing $\sigma=\lambda \theta_{1}+\mu \theta_{2}$ and computing gives

$$
\left\langle N\left(\sigma_{J}\right), \sigma_{J}\right\rangle_{f}=\frac{1}{2 \tau}\left\|\sigma_{J}\right\|^{2}>0
$$

and the theorem follows.

Corollary 1.2 follows immediately from this theorem.

Remark 4.4. In the recent work [5] the authors consider the operator

$$
\hat{\mathcal{L}}=\frac{1}{2} \Delta_{f}+R m(h, \cdot) .
$$

They prove that the Ricci form $\rho$ is twisted harmonic and that it satisfies $\hat{\mathcal{L}}($ Ric $)=$ $\frac{1}{2 \tau}$ Ric. Our proof here can be phrased as showing that the multiplicity of this eigenspace is at least $\operatorname{dim} H^{(1,1)}(M)$. One may then apply their Proposition 3.1 to conclude that Kähler-Ricci solitons are linearly unstable.

It would be most interesting to determine whether the perturbations $\sigma_{J}$ are in fact eigentensors of the operator $N$. It is clear that if $v_{\sigma_{J}}=0$, then $\sigma_{J}$ is an eigentensor. We observe that the recent work of Futaki-Sano [6] and Ma [8] gives a spectral gap for the Bakry-Émery Laplacian when the Bakry-Émery Ricci curvature is bounded below. They show:

Theorem 4.5 (Ma, Futaki-Sano). Let $\phi$ be a smooth function on $M$ and suppose that the Bakry-Émery Ricci curvature, defined as

$$
\operatorname{Ric}_{\phi}=\operatorname{Ric}(g)+\operatorname{Hess}(\phi),
$$

satisfies the bound

$$
\operatorname{Ric}_{\phi} \geq c,
$$

for some number $c>0$. Then the first non-zero eigenvalue $\lambda_{1}$ of the Bakry-Émery Laplacian $\Delta_{\phi}:=\Delta-\nabla_{\nabla \phi}$ satisfies

$$
\lambda_{1} \leq-c .
$$

Clearly this bound is weaker than the Lichnerowicz bound in the Einstein case. Hence one may not deduce that $v_{\sigma_{J}}=0$ for the unstable variations of Theorem 1.1 However, if $v_{\sigma_{J}} \neq 0$, such a soliton would prove that the above estimate is in some sense sharp. We suspect however that $v_{\sigma_{j}}=0$ and hence that these variations are eigentensors of $N$. Implicit in the calculation of the second variation formula is the fact that the potential function $f$ (once normalised) is an eigenfunction of $\Delta_{f}$ with

eigenvalue $-\frac{1}{\tau}$ (Futaki and Sano also prove this). It would be interesting to know if $\Delta_{f}$ has any eigenvalues in the interval $\left(-\frac{1}{\tau},-\frac{1}{2 \tau}\right]$, and the authors are currently numerically investigating this problem for the Koiso-Cao and Wang-Zhu solitons.

\section{REFERENCES}

[1] Huai-Dong Cao, Existence of gradient Kähler-Ricci solitons, Elliptic and parabolic methods in geometry (Minneapolis, MN, 1994), A K Peters, Wellesley, MA, 1996, pp. 1-16. MR1417944 (98a:53058)

[2] _ Geometry of Ricci solitons, Chinese Ann. Math. Ser. B 27 (2006), no. 2, 121-142. MR2243675 (2007f:53036)

[3] - Recent advances in geometric analysis, Adv. Lect. Math. (A.L.M.) 11 (2009), 1-38.

[4] Huai-Dong Cao, Richard S. Hamilton, and Tom Ilmanen, Gaussian densities and stability for some Ricci solitons, arXiv:math/0404165 (2004). 
[5] Huai-Dong Cao and Meng Zhu, On second variation of Perelman's Ricci shrinker entropy, arXiv:1008.0842v1 (2010).

[6] Akito Futaki and Yuji Sano, Lower diameter bounds for compact shrinking Ricci solitons, arXiv:1007.1759 (2010).

[7] Norihito Koiso, On rotationally symmetric Hamilton's equation for Kähler-Einstein metrics, Recent topics in differential and analytic geometry, Adv. Stud. Pure Math., vol. 18, Academic Press, Boston, MA, 1990, pp. 327-337. MR.1145263 (93d:53057)

[8] Li Ma, Eigenvalue estimates and L1 energy on closed manifolds, arXiv:0911.4828v1 (2009).

[9] Grisha Perelman, The entropy formula for the Ricci flow and its geometric applications, arXiv:0211159 (2002).

[10] Gang Tian and Xiaohua Zhu, Perelman's w-functional and stability of Kähler-Ricci flow, arXiv:0801.3504v1 (2008).

[11] Peter Topping, Lectures on the Ricci flow, London Mathematical Society Lecture Note Series, vol. 325, Cambridge University Press, Cambridge, 2006. MR2265040 (2007h:53105)

[12] Xu-Jia Wang and Xiaohua Zhu, Kähler-Ricci solitons on toric manifolds with positive first Chern class, Adv. Math. 188 (2004), no. 1, 87-103. MR2084775 (2005d:53074)

[13] Edward Witten, Supersymmetry and Morse theory, J. Differential Geom. 17 (1982), no. 4, 661-692 (1983). MR683171 (84b:58111)

Department of Mathematics, Imperial College, London, SW7 2AZ, United Kingdom

E-mail address: stuart.hallo6@imperial.ac.uk

School of Mathematical Sciences, University College Cork, Ireland

E-mail address: tommy.murphy@ucc.ie 